(RESEARCH ARTICLE)

\title{
Nutrition base of the anti-doping products development for the athletes working efficiency encreasment
}

\author{
Emil Muchamejanov *, Anatoliy Kulnasarov, Seilchan Karakov, Dias Shakenov, Victoria Belyavskaya \\ RSBSC Olympic Reserve named after K.Akhmetov, Kazakhstan, Almaty.
}

Publication history: Received on 20 April 2020; accepted on 29 April 2020

Article DOI: https://doi.org/10.30574/wjarr.2020.6.1.0109

\begin{abstract}
The aim of the article is to consider the metabolic basis of nutrition for the development of technologies to maintain the athlete's health and performance. The article used PubMed data and the results of our own theoretical developments regarding the metabolic basis of nutrition in extreme conditions of the body. The role of the stages of transcription and translation in the process of creating muscle protein and the importance of the quality and quantity of protein in the diet are shown. The anaerobic and aerobic stages of energy formation are examined and the principles of increasing their activity through the use of food compounds are analyzed. A model of the relationship between the exchange of proteins, fats and carbohydrates with different energy supply of the body is considered and the principles of nutritional support of the body in the phase of rehabilitation and physical and mental work are proposed. The article discusses the main approaches of the usage of metabolic principles in the development of food technology to maintain health and increase the working efficiency of athletes. In the sports preparation stage, nutrition should ensure the creation of a muscle apparatus and provide it with energy. In the sports competition period, food products should improve the activity of elements of energy metabolism. In the rehabilitation phase, it is necessary to use food compounds that help to reduce the accumulation of under-oxidized metabolic products and accelerate their metabolic pathways. Food products are offered to increase the effectiveness of weight gain and weight cutting technologies.
\end{abstract}

Keywords: Nutrition; Anti-doping products; Performance

\section{Introduction}

High - quality sport is associated with the maximum inclusion of all physiological systems, all types of metabolism. Therefore, there is a high risk of developing disorders of the cardiac, respiratory, thermoregulatory and hormonal systems [1]. The development of oxidative stress, the fall of the energy sources and the accumulation of harmful metabolites are noted [2], therefore, the urgent question is the development of health-saving technologies in which the nutritional factor plays a key role.

An athlete needs much more constructing (protein), energetic (carbohydrates and fats) and regulatory (vitamins and minerals) materials. It creates a problem to chew and absorb a large amount of food and athletes are at high risk of developing gastrointestinal tract disorders Therefore, there are many specialized products or functional nutrition products that appeared to provide the body with the easily digestible macro and micronutrients.

The main attention in scientific articles and monographs on the nutrition of athletes is paid to the issues of food hygiene - adequacy and safety. Of course, these aspects are important, but the question of the metabolic base of an athlete's nutrition has remained aside. Metabolism is an important aspect of maintaining health and working efficiency and the physical capabilities of a person depend on how metabolic processes proceed.

\footnotetext{
${ }^{*}$ Corresponding author: E Muchamejanov
} 


\section{Material and methods}

The article used PubMed data and the results of our own theoretical developments regarding the metabolic basis of nutrition in extreme conditions of the body.

\section{Results and discussion}

The athlete's nutrition should be divided into several stages:

- Nutrition at the preparation stage

- Nutrition for the energy supply of the competition period

- Nutrition to improve rehabilitation processes after intense physical activity

- Nutrition aimed to increase the effectiveness of technology for gaining or cutting weight.

The main task of the preparation stage is to create a muscle apparatus and provide it with energy. The main component of muscle fiber is a protein, so it is necessary to ensure its adequate supply [3]. The protein intake rate for a person is about $1 \mathrm{~g}$ per kg on body weight per day, whereas for an athlete, protein requires 30-50\% more.

Skeletal muscle, which is the most abundant tissue in the body of mammals, has a wide variety of important functions [5]. Skeletal muscle mass is determined by the dynamic balance between protein synthesis and degradation [6]. Skeletal muscle represents $40-50 \%$ of body mass in mammals and is a critical regulator of overall metabolism [7]. The protein synthesis of skeletal muscle has been proven to be affected by different nutritional and physiological factors [8-9]. The rate of protein synthesis and its type depend both on the activation of the genetic apparatus at the stage of transcription or genes expression [10], and at the stage of translation [11]. The mammalian target of the rapamycin (mTOR) signaling pathway is a central mediator of metabolism and growth and acts as a central regulator of protein metabolism [12].

Therefore, protein synthesis depends both on the availability of the protein synthesis process in the substrate, and on the protein synthesizing apparatus working efficiency, especially at the stage of translation. Protein synthesis occurs on ribosomes and is accelerated during aggregation of ribosomes into polysomes; therefore, factors that contribute to aggregation of ribosomes (acceleration of protein synthesis) and, conversely, their reverse decomposition into ribosomes (decrease in protein synthesis) should be considered, which is shown in Figure 1.

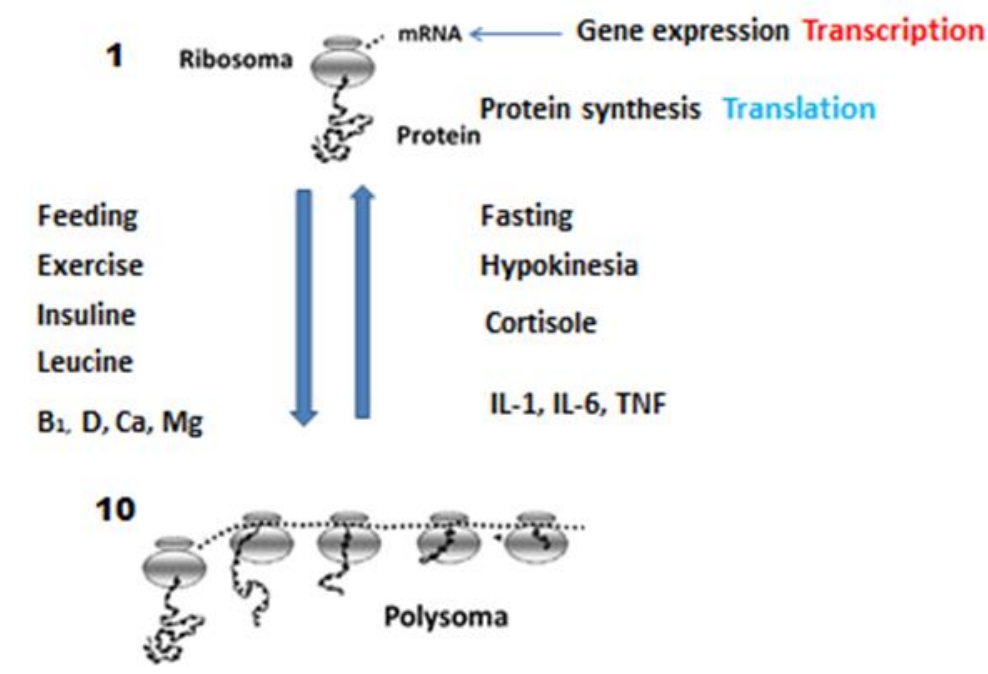

Figure 1 Regulation of the protein synthesis process at the stage of translation

After gene expression (transcription), mRNA enters the ribosome and a specific protein is synthesized. However, when ribosome aggregation to the polysome occurs, the mRNA passes through the entire aggregate and one mRNA promotes the synthesis of several proteins (corresponding to the number of ribosomes in the polysome). Therefore, if the amount of protein synthesis on the ribosome is conventionally taken as unity, then aggregation into the polysome increases the rate of protein synthesis dramatically. The factors for aggregating ribosomes or accelerating the process of protein synthesis are indicated on the left: 1) feeding or providing the process of protein synthesis with substrate and energy; 
2) physical activity or anabolic stimulus; 3) anabolic hormones, in particular insulin; 4) anabolic amino acids (leucine); 4) some vitamins and minerals (B1, D, Ca, Mg). These aspects must be considered when developing specialized foods to increase muscle mass.

On the right there are factors contributing to the disaggregation of polysomes and a decrease in protein synthesis: 1) starvation or when the substrate and energy are not enough for the protein synthesis process; 2) hypokinesia, which is a stress factor for the body; 3) cortisol or stress hormone; 4) inflammatory cytokines or processes where energy must be directed to solve the problem of inflammation. This situation occurs due to the fact that the body can afford to bear large costs for the process of protein synthesis only at rest, and during the development of energy stress, the body forced to direct all its efforts to eliminate it.

The second important aspect of sports is the energy provision of physical activity. There are several steps in the process of energy production that should be considered.

A lactate, when there is a creatine phosphate as an energy source. The 'anaerobic' substrates are also important fuels during the transition from rest to steady state exercise [13]. Although its energy is enough for only a few seconds of muscle work, creatine phosphate is located near the contractile elements and there is no need for the special transport systems for its delivery [14].

Lactate period or glycolysis. Due to glycolysis, the muscle can work up to 30 minutes. This is the only form of energy production of organic compounds that occurs under anaerobic conditions. Although only $7 \%$ of the energy of chemical bonds of glucose or 2 ATP is formed at this stage, glycolysis is characterized by a high rate of energy generation [15]. Since sport primarily is a speed, glycolysis is a key aspect of energy production for athletes.

The aerobic stage or the organic substances oxidation in the cycle of tricarboxylic acids and the ATP energy formation in the process of oxidative phosphorylation.

At this stage, $93 \%$ of glucose energy and $100 \%$ of fat energy are generated. In quantitative terms, this is the main way of energy formation and it determines the duration or persistence of muscle activity. Therefore, questions related to which ways we can influence the stages of energy formation should be considered.

Creatine phosphate is formed by creatine phosphorylation that is a tripeptide (glycine, arginine, and methionine) [16]. Glycine is a nonessential amino acid, arginine is a conditionally nonessential amino acid, because the need for it increases with intensive growth in children and during muscle gaining. The limiting amino acid is methionine. Methionine, found almost exclusively in skeletal muscle, results in creatine levels that can be increased via endogenous synthesis in the liver and pancreas or exogenously from foodstuff, particularly meat and fish [17]. Creatine preparations began to be widely used after the Olympic Games in 1992 in Barcelona [18]. In specialized diets, its content does not exceed 1-2 g [19], while in a young man weighing $70 \mathrm{~kg}$ the pool of creatine is $120-140 \mathrm{~g}$. Therefore, in the preparation stage, you should use creatine preparations.

Hydrolysis of creatine phosphate promotes ATP muscle regeneration.

Creatine phosphate + ADP $\longrightarrow$ ADP + Creatine

However, creatine formed during the hydrolysis of creatine phosphate attaches water and turns into creatinine, which is removed from the blood into the urine due to glomerular filtration. Creatinine formation depends on the amount of muscle mass; therefore, its content in urine correlates with the value of muscle or lean mass [20]. In case of impaired renal function, creatinine excretion from blood to urine decreases and blood/urine creatinine coefficient increases, which is used in the clinic to evaluate kidney function. In sports practice, it is more convenient to use a non-invasive method for assessing renal function by salivary/urine creatinine coefficient [21].

Glycolysis depends on the availability of glucose in the body. There a lot of problems. Glucose is the main source of energy and presents at the intersection of many metabolic processes, so its level should be maintained at the homeostatic level. The blood contains only about $5 \mathrm{~g}$ of glucose. This is easy to estimate by calculation. Normally, the blood glucose is $100 \mathrm{mg} \%$ (100 mg per $100 \mathrm{ml}$ ) [22], the body contains about 5 liters of blood, therefore, the total amount of glucose in the blood is $5 \mathrm{~g}$. With a decrease of the concentration of glucose in the blood twice lower than normal in the brain there will be insufficient glucose (the brain uses glucose exclusively as an energy source) and brain will turn off (instant loss of consciousness), and after 5 minutes the brain cells will die and death will occur [23]. 
In fact, during usage of blood glucose, glucose instantly replenishes due to liver glycogen. Although the total glycogen content in muscles is almost 5 times higher, but in the muscles there is no glucose-6-phosphatase enzyme, therefore muscle glycogen cannot be a source of free glucose. This was created by nature for the purpose that muscle glycogen can be used only by the muscle itself or if the animal or in particular the person does not catch up their food, they will simply die because of starvation.

Therefore, the maintenance of glucose homeostasis is an important aspect of the life process and all types of metabolism, all regulatory systems are involved in the regulation of glucose homeostasis. There are several sensory systems for controlling glucose homeostasis in the body and cells. When glucose enters the cell from the blood, the phosphorylation of glucose with the participation of the hexokinase enzyme occurs and glucose-6-phosphate (G-6-F) is formed. Therefore, hexokinase is the first sensor to control glucose metabolism. According to the feedback principle, G-6-F is an inhibitor of hexokinase, therefore, when the rate of conversion of G-6-F is reduced, the flow of glucose into the muscle cell is inhibited. This is observed with a decrease of ATP energy utilization, which leads to an increase in the ATP/ADP coefficient and inhibition of hexokinase [24].

During intense physical exertion, the rate of energy consumption exceeds the amount of G-6-F oxidation, therefore ATP resynthesize is also carried out due to the phosphate group of ADP, which leads to the formation of AMP, which is a sensor of insufficient glucose in energy production processes, therefore, the AMP/ATP coefficient characterizes degree of energy stress. Under energy stress, AMP binds to the $\gamma$ subunit of AMP dependent protein kinase (AMPK), its activity is allosterically activated, which leads to the suppression of protein anabolism, i.e. the body disables the stage of energy expenditure on protein synthesis [25]. Therefore, AMPK is a sensor for monitoring glucose homeostasis.

Based on the ways of transporting the carbon skeleton and the stages of the relationship between the processes of formation and utilization of ATP energy with different supply of glucose to the body, a conceptual model of the relationship between the exchange of macronutrients (proteins, fats and carbohydrates) was developed to maintain glucose homeostasis (Fig. 2).

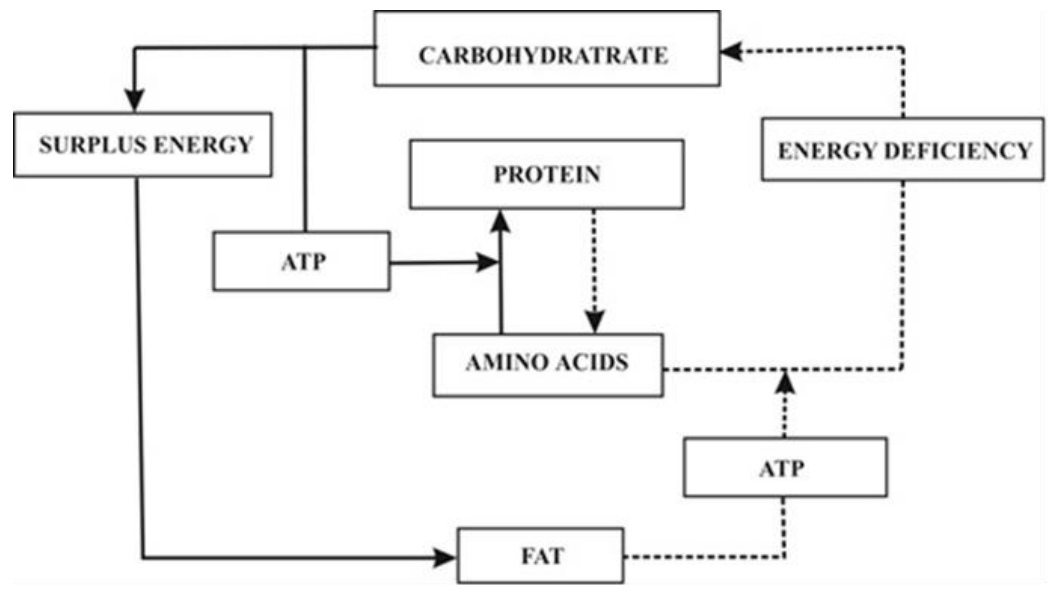

Figure 2 A conceptual model of the relationship between metabolism of protein, fat and carbohydrates for utilization exogenous $(\rightarrow$ ) and endogenous $(. . . \rightarrow$.) nutrition flow.

During the "Surplus energy" (for utilization exogenous nutrition flow) glucose utilization (dissimilation) is accomplished via storing it as glycogen and oxidation. If glucose inflow exceeds its dissimilation rate, then the excess carbon skeleton is utilized for the synthesis of lipids, that is, a combined anabolic lipogenesis is switched on.

Glucose oxidation results in the generation of ATP energy, therefore, there should exist routes to utilize this energy, since the excess ATP automatically block glycolysis. One may consider three principal ways of utilization of ATP energy: expenditures for physical activity, expenditures for heat production, and utilization for protein synthesis. In fact, physical activity, increased heat production and increased protein intake result in acceleration of glucose utilization. From the dietetic standpoint the process of protein synthesis is of interest. In other words, the rate of amino acids intake with food is an important regulator of glucose utilization.

Thus, during the "Surplus energy" the process of glucose dissimilation is associated with the two assimilation processes: with lipogenesis in regard of carbon skeleton, and with protein synthesis in regard of generation and utilization of ATP energy. 
Even though glycolysis and protein synthesis are interconnected via generation and utilization of ATP energy, however, these metabolic flows are closely interrelated since no protein synthesis occurs without energy supply while reduced utilization of ATP energy blocks ATP generation or glycolysis. In such case an excess carbon skeleton will be redirected to lipid synthesis resulting in obesity.

During the "Energy deficiency" (for utilization endogenous nutrition flow) glucose homeostasis is maintained on the account of its endogenic synthesis from amino acids, that results in protein catabolism to supply the required substrates while lipolysis and lipid oxidation get activated to supply the energy for gluconeogenesis. This stage is characterized with combination of two dissimilation processes (protein catabolism and lipid oxidation) and one assimilation process (gluconeogenesis). Glucose synthesis is associated with lipid oxidation through the generation and utilization of ATP energy, while with protein catabolism - via routes of transportation of carbon skeleton.

Though gluconeogenesis and lipid oxidation are associated with each other through the generation and utilization of ATP energy, these metabolic flows are inter-dependent. For example, blockade of lipolysis [26] or lipid oxidation [27] automatically causes the decline of gluconeogenesis resulting in hypoglycemia, and on the contrary, the reduction of concentration of the substrate for gluconeogenesis blocks ATP synthesis from acetyl-CoA and results in condensing of excess acetyl groups in acetoacetate and oxybutyrate, leading to ketosis, for instance, in diabetes or fasting [28].

Thus, glucose homeostasis in the body depends to considerable extent on interrelations between the metabolism of proteins, lipids and carbohydrates. This dependence is determined by the capacity of any component of the food to affect individual steps of conversion of other nutrients with involvement of regulatory function of hormones. This model may serve as a theoretical basis to develop a dynamic model of balanced nutrition.

Models of glucose recycling via lactate or alanine are circular, that is, carbon skeleton of glucose is used to synthesize lactate or alanine while glucose synthesis requires the return of carbon skeleton. In our model during the "Surplus energy" the carbon skeleton of glucose is used for lipid synthesis while at the "Energy deficiency" there occurs a loss of that carbon skeleton (elimination in the form of carbon dioxide at lipid oxidation). On the contrary, ATP energy generated at glucose oxidation is spent for protein synthesis, and we again get back the carbon skeleton.

Hence, our model can be defined as a cross-like one, since there is no direct route of transportation of carbon skeleton or energy because of opposite direction of assimilation and dissimilation of proteins, lipids and carbohydrates during the "Surplus energy" and the "Energy deficiency". If during the "Surplus energy" one observes activation of protein and lipid assimilation and carbohydrate dissimilation, then during the "Energy deficiency" protein and lipid dissimilation and carbohydrate assimilation occur, thus glucose homeostasis in the body is maintained on account of utilization its excess flow to include the carbon skeleton in lipid synthesis, while ATP energy is directed to protein synthesis, and during the endogenic glucose synthesis lipids provide ATP energy while proteins supply the carbon skeleton.

Metabolism intensity is controlled by neuro-endocrine system. The "Surplus energy" is signaled by acetylcholine and insulin levels while the "Energy deficiency" is mediated through noradrenaline and glucagon levels. Therefore, on the one hand, the neuro-hormonal status reflects energy balance of the body, and on the other hand, it depends on the intensity and ratio of nutrient flows.

Extensive studies on the specifics of metabolism in fasting or intake of individual nutrients are available; therefore these states are a convenient model to assess the intensity of metabolic flows from the position of the proposed model. Hepatic glycogen stores almost completely disappear after a 24-48 hour fasting [29-30], therefore the body is supplied with glucose due to protein catabolism [31] and lipid oxidation. Introduction of the key gluconeogenic amino acid alanine causes an increased glucose production in the liver [32] while oleic acid (energy substrate for gluconeogenesis) increases hepatic glucose production almost two fold [33], and on the contrary, the inhibition of lipolysis [34] or fatty acid oxidation [35] result in hypoglycemia.

Thus, metabolic changes arising at various physiological states of the body and metabolic disorders are determined to a considerable extent by inadequacies between nutrient flows that stipulate the possibility of correcting them with nutritional factors.

A certain balance between individual nutrient flows should be maintained. During the "Surplus energy" such balance should be met between the flows of glucose and amino acids. Excess glucose flow induces hyperglycemia and lipidemia while inadequate glucose intake with food leads to a lower inclusion of amino acids in proteins resulting in hyperaminoacidemia. Therefore, adequacy between these nutrient flows is the most important principle of balanced nutrition. 
During the "Energy deficiency" depot flows are utilized. The balance between the flows of amino acids (pool of free amino acids) and lipids is of key importance at this stage. It is well known, that lipid reserves (in the form of triglycerides) are much higher than protein reserves (in the form of muscular protein), and therefore the prevalence of lipid flow is the most often seen phenomenon. This inadequacy becomes more evident in low-protein rations when there are no enough amino acids or energy for protein synthesis. In such cases there occurs the reduction of the pool of free amino acids and more pronounced hypoglycemia and ketosis in fasting. Hence, there take place dynamic changes of metabolic indices which depend on the ratio of individual nutrients in food; therefore, the proposed model is a theoretical basis for the dynamic model of balanced nutrition.

For the formation of a peptide bond (a combination of two amino acids), 3 ATPs are used (fee for accuracy and speed), the average protein consists of 100 peptide bonds, thousands of proteins are synthesized in the body per day, therefore protein synthesis is the most energy-intensive process and can only be carried out in parallel with the process the formation of energy or a concomitant catabolic process. Only glucose can act in such process in the absorptive period (after eating), when the hormone insulin is released during food, which inhibits the oxidation of fats and, accordingly, the ability to use fats as an energy source is turned off. Therefore, between the process of protein synthesis and glucose oxidation, a correlation is revealed at the level of utilization and generation of ATP energy.

This aspect can be applied to develop a personalized approach for creating a diet for an athlete. If an athlete is given a standard breakfast with a known ratio of proteins, fats and carbohydrates and after 30-60 minutes to evaluate the level of glucose and amino acids in the blood, then excessive manifestations of glycaemia will indicate that there is an excess of carbohydrates and a protein deficiency in his diet. With manifestations of hyperaminoacidemia, an inverse relationship is noted or the diet contains an excess of protein and not enough carbohydrates. These manifestations may depend on the individual (metabolic) characteristics of the athlete, on seasonal influences and the influence of various environmental factors, which can be corrected by changing the ratio of carbohydrates or protein in the diet. In this way, it is possible to reduce the negative manifestation of metabolic disorders and increase the effectiveness of the preparation stage in relation to increasing the athlete's working efficiency.

In the sports competition period, the most important task is to provide motor activity with energy sources and reduce the negative impact of the excessive formation of under-oxidized products of the exchange of glucose (lactic acid) and amino acids (ammonia). During glycolysis, 6 carbon glucose molecules are split into two parts (Fig. 3).

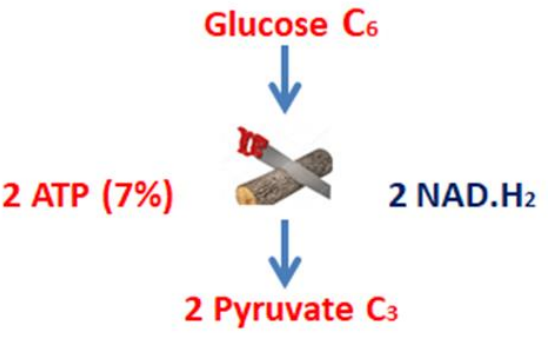

Figure 3 Schematic representation of the stage of glycolysis

As can be seen from Figure 3, when glucose is oxidized during the glycolysis step, 2 ATP molecules are released, as well as 2 hydrogen $\left(\mathrm{H}_{2}\right)$ molecules, which are not in gaseous form, but in connection with the organic molecule nicotinamide dinucleotide (NAD.H $_{2}$. If ATP is used for physical activity, then hydrogen must be combined with oxygen in the process of biological oxidation (phosphorylation), which occurs in mitochondria. However, the mitochondrial membrane is not permeable to NAD.H2, therefore, a transporter is needed, which is a malate-aspartate shunt, the limiting component of which is the aspartic amino acid (aspartate), so intake of aspartate helps to increase physical efficiency [36].

With aspartate deficiency, the amount of NAD. $\mathrm{H}_{2}$ in the mitochondria decreases, which leads to an increase in the NAD.H $H_{2}$ NAD coefficient in the cytoplasm or oxidation/reduction reactions are disturbed, which poses a threat to vital processes. Therefore, it is necessary to "dump" hydrogen (reduction equivalent) onto the oxidized compound, in particular pyruvate, which leads to an increase in the formation of lactic acid (lactate). In fact, the level of lactate is a reflection of the degree of impairment of oxidation - reduction reactions, and when it enters the brain, it is a sensor of these disorders. Therefore, to maintain vital activity, the brain during an alarming level of lactate, gives the command to stop physical activity through signals of fatigue or refusal to work.

The accumulation of lactate in the muscles contributes to the development of acidosis and impaired contractility of the muscles. Muscle damage and oxidative stress are other causes of fatigue that could be minimized by glutamine. In the 
skeletal muscle, glutamine comprises $50-60 \%$ of the total free amino acid pool, being considered as the most synthesized amino acid in the human muscle, especially in slow-twitch muscles, which contain glutamine concentrations 3-fold higher than fast-twitch muscles [37]. Showed that glutamine supplementation (for 21 days) reduced the plasma concentrations of creatine kinase (CK) and lactate dehydrogenase (LDH) - markers of muscle damage in rats submitted to strenuous resistance training [38]. The dipeptide carnosine (beta-alanine and histidine), which contains in the muscles, acts as a buffer against muscle acidosis. Histidine is an essential amino acid, therefore, the synthesis of carnosine depends on the availability of beta-alanine in the body and there are facts about the positive effect of beta-alanine on the athlete's performance [39].

Pyruvate can be converted in several ways (Fig. 4).

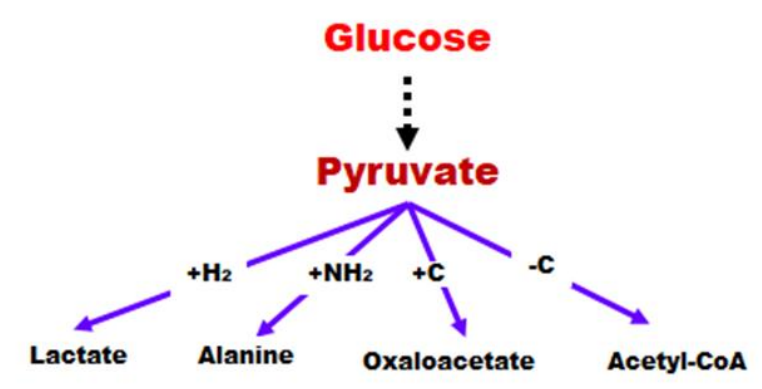

Figure 4 Pathways for the conversion of pyruvate

With the participation of Lactate dehydrogenase, pyruvate is converted into lactic acid (lactate);

With the participation of Alanine aminotransferase, pyruvate is converted to the amino acid alanine;

With the participation of Pyruvate carboxylase, pyruvate is converted to oxaloacetate;

With the participation of Pyruvate dehydrogenase, pyruvate is converted to Acetyl-CoA.

As was mentioned earlier, compounds at the crossroads of metabolic pathways must be maintained at the homeostatic level, therefore, a violation of the conversion of pyruvate along one of the pathways will lead to its pathway formation block or glycolysis ("pyruvate block").

Therefore, by improving pyruvate metabolism, we will reduce the manifestations of the pyruvate block and accelerate the glycolysis process or increase the athlete's working efficiency.

As mentioned earlier, the conversion of pyruvate to lactate contributes to a decrease of efficiency, therefore, under anaerobic conditions, it is preferable to conversion pyruvate to alanine, because this reduces the magnitude of lactic acidosis and decreases impaired muscle contractility. For the transamination of pyruvate to alanine, amino groups are required, which are branched chain amino acids (BCAAs), in particular leucine, as donors. These amino acids are considered as anabolic and are recommended for muscle building, but if it taken before exercise, it increases the muscle pool and the possibility of their use for amination of pyruvate, the manifestations of lactic acidosis and the development of the "pyruvate block" will decrease [40].

The concentration of lactate in the blood depends on both the size of its formation and the rate of its utilization. In this regard, it is possible to promote an increase the rate of its utilization using the "metabolic training" technique. In 1962, Corey proposed a model of lactate recycling (41), when the lactate stream is used as a substrate for the reverse synthesis of glucose (gluconeogenesis). The activity of gluconeogenesis enzymes depends on their expression, which can be enhanced by the use of a fermented milk product. However, during fermented milk fermentation both L and D forms of lactate form, however L-lactate is biologically active. Therefore, a strain of lactic acid bacteria was selected that synthesized predominantly the L form of lactate. This dairy product was named "Lactan" (derived from the words lact - milk and an - anaerobic). This product was given to the USSR women's speed skater team (trainer B.Stenin) for two weeks in amount of 0.5 liters during their training session at the Medeo ice rink. The product was given at bedtime, which was an additional training for athletes to increase the metabolic capacity for lactate utilization. At the beginning and at the end of the training camp, the standard "pyramid" exercise was carried out, when speed skaters ran a circle at a certain speed, then rest for 2 minutes, then run two circles, again rest 2 minutes, then 3 circles and rest 2 minutes and finally 4 circles and rest 2 minutes. Then the total load was reduced, i.e. running of 3 circle and again rest 2 minutes, then 2 circles and rest 2 minutes and at the end 1 circle. After that, blood was taken from the skaters and 
the level of lactate was estimated, which on the average was $60 \mathrm{mg} \%$ through the group. At the end of the training session, the exact same load was performed and lactate concentration was measured again and it amounted to $32 \mathrm{mg} \%$ (unpublished data). The trainer noted an improvement of the training process and high personal indications during sprint speed skating competitions (500 and 1000 meters). The intake of Lactan can be attributed to the technology of "food hypoxia", which is very easy to use and can replace high altitude exposure technologies or the use of gas mixtures. The use of reconstructed compounds, in particular succinate, was widely promoted by bioenergy in the $80 \mathrm{~s}$ of the last century. However, their ideological postulate was based on the fact that for the oxidation in the cycle of tricarboxylic acids for acetyl-CoA four stages of dehydrogenation are required, but for succinate only two, i.e. it is a faster energy generation system. However, only a certain amount of substrate may be contained in the cycle, and the excess flow of succinate will simply be "discharged" into fats (activates lipogenesis). However, the reconstructed equivalents contain in succinate contribute to the activation of utilization systems and thereby increase efficiency.

Under aerobic conditions, pyruvate can add carbon (carboxylate to oxaloacetate) or release carbon (decarboxylate to acetyl $\mathrm{CoA}$ ). Vitamin $\mathrm{B}_{1}$, magnesium, lipoic acid are involved as cofactors in carboxylase and pyruvate dehydrogenase activities; therefore, their deficiency impairs the activity of these enzymes, decreases the amount of pyruvate utilization, and develops a pyruvate block [42-45].

When acetyl-CoA enters the mitochondria, it combines with oxaloacetate (OA) to form citric acid, so the possibility of the interaction of acetyl-CoA with OA will depend on the amount of OA available. With simultaneous operation, there is a correspondence between the amount of acetyl-CoA formation and the presence of OA in the cycle. If it is necessary to enhance the generation of energy or increase the flow of acetyl-CoA into the mitochondria, also it is necessary to simultaneously increase the content of OA there. OA can be formed from aspartic acid, but using amino acids is an expensive pleasure, so OA is formed mainly during the carboxylation of pyruvate glucose metabolite. Therefore, any changes in the rate of energy production are possible only due to changes in the rate of formation of OA or glucose utilization. The most demonstrative sprinter in humans is the brain, because the processes of excitation/inhibition are carried out during seconds, and such changes in the process of energy formation can be carried out only due to glucose. Therefore, the brain, with its most powerful oxidizing system, can only use glucose as an energy source. Of course, the brain can switch to using fatty acids as an energy source, but only in the case of inhibition of brain activity, for example during anesthesia, or coma. For tactical purposes, the athlete must periodically change the rate of energy production, which is ensured only by glucose, whose reserves in the body are limited. Dietary manipulations are an integral part of an athletes training process, related significantly to optimal performance. The mechanisms responsible for improved working efficiency are best recognized for high-carbohydrate diets, and are attributed to maximizing muscle glycogen content and thereby its availability and utilization during exercise [46].

According to our proposed model of the relationship between the exchange of proteins, fats and carbohydrates, two food streams should be considered: absorbent or exogenous and postabsorbtive or endogenous. By the content of macronutrients, they differ significantly (Fig. 5).

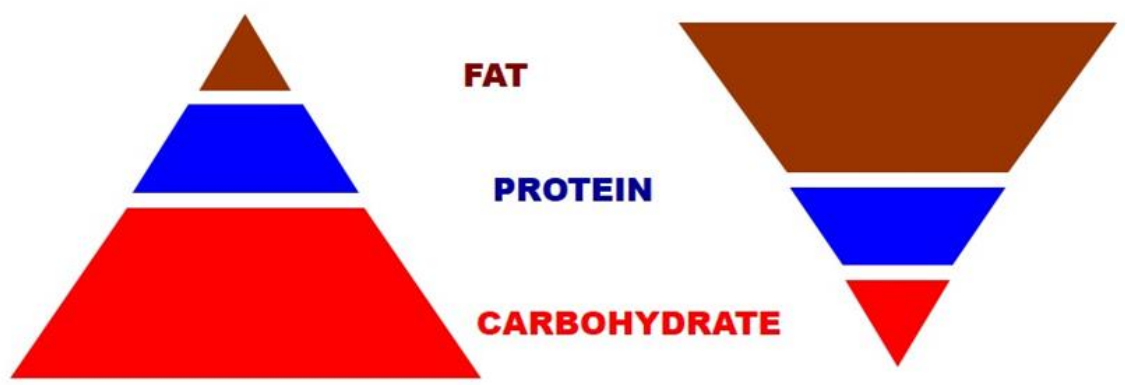

Figure 5 The ratio between proteins, fats and carbohydrates in exogenous (left) and endogenous (right) food streams the form of pyramids

In the absorptive period (exogenous food stream), carbohydrates are the main macronutrient, therefore exogenous nutrition is highly carbohydrate. There is no glucose in the muscle itself, because during passing through the cell membrane it turns into G-6-F. Upon further conversion of G-6-F, another ATP molecule is expended. The body wouldn't just waste ATP energy, and phosphorylation is carried out to connect sensory systems for monitoring glucose metabolism [47].

The intake of glucose before a training or competition process will contribute to the secretion of insulin and the activation of protein synthesis and the deposition of excess energy flows in the form of glycogen and lipids, but this will 
lead to a decrease of working efficiency ("a well-fed animal is not a hunter"), therefore, in all recommendations there is a warn not to eat before exercise.

During the period of physical work, endogenous food stream are used and, as can be seen from Figure 5, fats are the main component, the oxidation of which is inhibited by insulin [48]. During this period, food compounds for which insulin secretion does not occur can be used. Based on such considerations, we created a product that included artichoke and palm oil. An English patent has been obtained for that product (49). Although it was created in order to maintain glucose homeostasis while reducing the diet of obese people, it can be used in sports to maintain glucose homeostasis during intense physical exertion.

The reconstructed equivalents formed during the dehydrogenation of organic compounds, during glycolysis and in the tricarboxylic acid cycle are included in the oxidative phosphorylation chain or the "slow combustion" process. The slow combustion differs from ordinary combustion in fact that energy is released by stages (three stages of breathing and phosphorylation). At the first stage of electron or hydrogen transfer, coenzyme $\mathrm{Q}_{10}\left(\mathrm{CoQ}_{10}\right)$ takes part. CoQ10 (ubiquinone) is a widespread component of human and animal tissues. The necessary components for the biosynthesis of ubiquinone are 4-hydroxybenzoate and polyprenyl chain [50]. The content of CoQ10 largely depends on its synthesis in the body [51]. With age, there is a decrease in the rate of CoQ10 synthesis [52] and a decrease in the rate of energy production under aerobic conditions. Q10 deficiency was observed in athletes with poor efficiency and in individuals with manifestations of encephalopathy and myopathy [53].The impairment of the antioxidant-mediated defense system during acute exercise training, which has been shown in muscles and the liver, results in the increase of reactive oxygen species (ROS) and inflammation markers [54]. At the end of the maximal exercise, this condition of the muscle tissue causes free radicals to multiply, leading to lipid peroxidation of the membranes, and an increase in the abundance of macrophages and white blood cells [55]. Exercise can increase the use of oxygen by over 200 times and increases the relaxation levels of working muscle fibers [56]. Increased amounts of free radicals associated with excess oxygen consumption is counteracted by a defense system containing enzymatic and non-enzymatic antioxidants. Excessive exercise is manifested as muscle fatigue and muscle damage known as oxidative stress due to imbalance between ROS and antioxidants [57]. Regular exercise provides many benefits, while excessive exercise increases oxidative damage by increasing the ROS formation [58]. Studies have been conducted on both marathon runners and experimental animals in order to eliminate or mitigate such negative effects caused by exercise. These studies were mostly based on the removal of oxidative stress and were conducted with reinforcing substances which are thought to have strong antioxidant properties.

They have many functions in maintaining intracellular integrity via protection, repair, and even regulation of cell death signaling [59]. Chronic exercise may improve the overall health; however, a single bout of inadequate exercise may lead to oxidative stress and muscle damage [60]. Taken together, these studies suggest that the expression of HSPs could be considered important for protection from and repair of skeletal muscle after exercise-induced stresses.

Coenzyme Q10, naturally found in mitochondria, is a potent antioxidant that is endogenously synthesized and is soluble in fat [61]. Due to its antioxidant properties, it is able to effectively inhibit the oxidation of fat, protein, and DNA in the body [62]. CoQ10 suppresses lipid peroxidation, and thus, oxidative stress by suppressing the activity of enzymes involved in ROS production [63]. One of the most important functions of CoQ10 is to serve as an electron carrier during oxidative phosphorylation by diffusing to the phospholipid layer of the cell membrane via a unique chain structure in mitochondria [64]. In addition, it plays an important role in energy production from carbohydrates and fatty acids in cells [65].

Oxidative stress, which occurs during extreme exercise, lowers CoQ10 levels in mitochondria [66]. Thus, excessive free radicals are created that accumulate in the muscles, leading to fatigue and muscle damage in the body [67]. It has been reported that $\mathrm{CoQ}_{10}$ supplementation is highly effective in transporting and using oxygen required at the cellular level [68] as well as in eliminating muscle damage and fatigue [69]. During CoQ10 deficiency in the muscles, lipid oxidation is impaired [70]. CoQ10, being an antioxidant, has an anti-inflammatory effect, is involved in DNA replication and repair (being an indispensable cofactor for the synthesis of pyrimidine bases), in the process of regulation of the physicochemical properties of cell membranes, and gene expression [71]. The intake of $\mathrm{Q}_{10}$ by rats reduces the damage caused by the load [72]. In the reconstructed form, $Q_{10}$ prevents lipid peroxidation, acting as an antioxidant [73], and prevents the development of oxidative disorders. Unlike conventional antioxidants, CoQ10 is included in the oxidation / reduction exchange (ubiquinone/ubiquinole) and promotes the work of the mitochondrial electron transfer shunt or presents not only as antioxidant, but also as prooxidant.

In this regard, it can be compared with the sulfated polysaccharide of brown seaweed fucoidan, which manifests both antioxidant [74] and prooxidant properties [75]. 
Fucoidan is a nutraceutical with a wide spectrum of bioactivity. It has been previously suggested that fucoidan has various biological activities such as antibacterial [76], antioxidant [77], anti-inflammatory [78], anticoagulant [79], therefore, its intake will contribute to maintaining health and increasing the efficiency of athletes.

Depending on the degree of physical activity, a V-shaped change in the growth of acute respiratory diseases (ARI) or upper respiratory tract infections is noted [80]. Excessive physical activity leads to a decrease of immunity and this is accompanied by an increase of the level of acute respiratory infections. The authors showed that in high - quality athletes after the marathon in $33 \%$ of cases (in the control only 15\%) the development of acute respiratory infections is observed, which correlates with the activity of B and T lymphocytes. In 11 high - quality marathon runner during taking fucoidan at a dose of 250 and $500 \mathrm{mg}$ [81] compared with the control (placebo), there was a decrease in the level of acute respiratory infections. In the control, the level of acute respiratory infections reached $17 \%$ two weeks after the race and $6 \%$ a month later. Giving fucoidan in a dose of $250 \mathrm{mg}$ contributed to a decrease of the frequency of acute respiratory infections to 8 and $2 \%$, and at a dose of $500 \mathrm{mg}$ to 6 and 2\% (the differences are significant). For another indicator (health assessment), a positive effect of fucoidan was also noted.

The positive effect of ingestion of food compounds on the prevention of acute respiratory infections in high- quality athletes has already been pointed out [82-84]. Intake of fucoidan in dose $5.6 \mathrm{~g} /$ day contributed to the restoration of the immune system [85].

A fucoidan dose $(0.31 \mathrm{~g} / \mathrm{kg})$ was used in an experiment with mouses, here was converted from a human equivalent dose (HED) based on body surface area by the following formula from the US Food and Drug Administration: assuming a human weight of $60 \mathrm{~kg}$, the HED for $1.5(\mathrm{~g}) \div 60(\mathrm{~kg})=0.025 \times 12.3=$ a mouse dose of $0.31 \mathrm{~g} / \mathrm{kg}$; the conversion coefficient 12.3 was used to account for differences in body surface area between a mouse and a human. An increase in muscle strength and fatigue resistance (forelimb), an improvement of biochemical parameters were noted [86].

In sports practice, for tactical reasons, it is necessary to change body weight, in particular, carry out the weight loss (cutting). In this case, lean or muscular body mass should be maintained. Amino acids are the main substrate for endogenous glucose synthesis, therefore, during the reduction of food intake for the purpose of maintain glucose homeostasis, the utilization of amino acids and protein catabolism are enhanced, which contributes to the development of various functional disorders that are well studied during prolonged fasting (Fig. 6).

\section{Health: $100 \%$ nitrogen cell body}

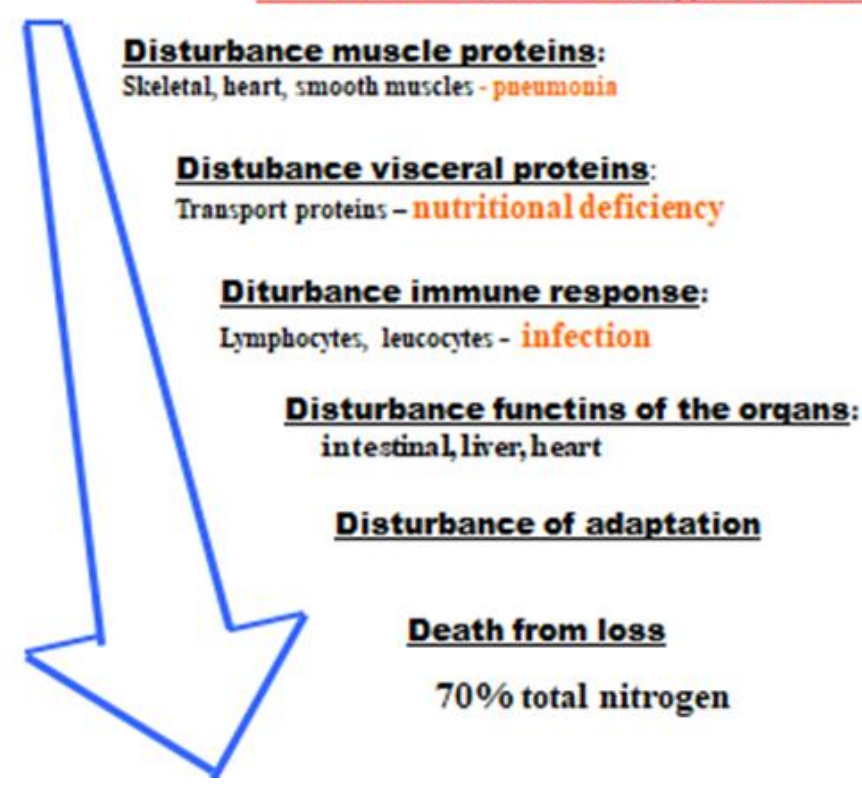

Figure 6 The development of functional disorders according to dynamics of fasting

In athletes, these manifestations are noted with excessive training. This is due to the fact that intense physical activity requires increased formation of the main energy source (glucose) or utilization of amino acids as a substrate.

Therefore, excessive physical activity leads to the utilization of muscle mass and the development of muscle weakness, the use of visceral proteins or blood proteins. This leads to a deterioration in the transport of food compounds and the 
development of manifestations of food deficiency. The use of blood cells (lymphocytes, monocytes) leads to a decrease of the immune response and the development of infections. Then there is a violation of the function of organs and tissues. Trainers often believe that the higher the training load, the higher the results, however, excessive physical activity contributes to reduced efficiency, because the manifestation of excessive training develops, but a coach believes that the athlete simply takes care of himself.

These disorders can be effectively neutralized by maintaining glucose homeostasis through the use of a specialized product that we developed, which we called "Sportop". In that case person can use excess physical activity. At the present time the formulation is upgrading to increase the effectiveness of the product for sports activities. The metabolic principles for the development of specialized foods for athletes will significantly enhance the functional aspects of such foods.

\section{Conclusion}

The article discusses the main approaches of the usage of metabolic principles in the development of food technology to maintain health and increase the working efficiency of athletes. In the sports preparation stage, nutrition should ensure the creation of a muscle apparatus and provide it with energy. In the sports competition period, food products should improve the activity of elements of energy metabolism. In the rehabilitation phase, it is necessary to use food compounds that help to reduce the accumulation of under-oxidized metabolic products and accelerate their metabolic pathways. Food products are offered to increase the effectiveness of weight gain and weight cutting technologies. Particular attention is paid to the use products with the inclusion of two perspective nutraceuticals - coenzyme Q10 and fucoidan in sports practice. The ability to improve the flow of metabolic processes with the help of food compounds is an anti-doping approach in case of maintaining health and increasing the athletes' efficiency.

\section{Compliance with ethical standards}

\section{Disclosure of conflict of interest}

Conflicts Authors declare that the research was conducted in the absence of any commercial or financial relationships that could be construed as a potential conflict of interest.

\section{References}

[1] Mitchell JH and Blomqvist G. (1971). Maximal oxygen uptake. N. Engl. J. Med, 284, 1018-1022.

[2] Reid MB. (2001). Nitric oxide, reactive oxygen species, and skeletal muscle contraction. Med. Sci. Sports Exerc, 33, 371-376.

[3] Wilson JP, Strauss BJ, Fan B, Duewer FW and Shepherd JA. (2013). Improved 4-compartment body-composition model for a clinically accessible measure of total body protein. Am J Clin Nutr., 97, 497-504.

[4] Phillips SM. (2012). Dietary protein requirements and adaptive advantages in athletes. Br J Nutr., 108, S158-S167.

[5] Fanzani A, Conraads VM, Penna F and Martinet W. (2012). Molecular and cellular mechanisms of skeletal muscle atrophy: an update. J Cachexia Sarcopenia Muscle, 3, 163-179.

[6] Gordon BS, Kelleher AR and Kimball SR. (2013). Regulation of muscle protein synthesis and the effects of catabolic states. Int J Biochem Cell Biol., 45, 2147-2157.

[7] Sandri M. (2010). Autophagy in skeletal muscle. FEBS Lett., 584, 1411-1416.

[8] Escobar J, Frank JW, Suryawan A, Nguyen HV, Kimball SR, Jefferson LS and Davis TA. (2005). Physiological rise in plasma leucine stimulates muscle protein synthesis in neonatal pigs by enhancing translation initiation factor activation. Am J Physiol Endocrinol Metab, 288, E914-E921.

[9] Bolster DR, Jefferson LS and Kimball SR. (2004). Regulation of protein synthesis associated with skeletal muscle hypertrophy by insulin-, amino acid- and exercise-induced signalling. Proc Nutr Soc., 63, 351-356.

[10] Jacques M, Hiam D, Craig J, Barrès R, Eynon N and Voisin S. (2019). Epigenetic changes in healthy human skeletal muscle following exercise- a systematic review. Epigenetics., 14, 633-648. 
[11] Welle S, Burgess K and Mehta S. (2009). Stimulation of skeletal muscle myofibrillar protein synthesis, p70 S6 kinase phosphorylation, and ribosomal protein S6 phosphorylation by inhibition of myostatin in mature mice. Am J Physiol Endocrinol Metab., 296, E567-E572.

[12] Dennis PB, Fumagalli S and Thomas G. (1999). Target of rapamycin (TOR): balancing the opposing forces of protein synthesis and degradation. Current Opinion in Genetics \& Development, 9, 49-54.

[13] Hargreaves M. (2000). Skeletal muscle metabolism during exercise in humans. Clin Exp Pharmacol Physiol., 27, 225-228.

[14] Yoshizaki K, Watari H and Radda GK. (1990). Role of phosphocreatine in energy transport in skeletal muscle of bullfrog studied by 31P-NMR. Biochim Biophys Acta, 1051, 144-150.

[15] Pfeiffer T, Schuster S and Bonhoeffer S. (2001). Cooperation and competition in the evolution of ATP-producing pathways. Science, 292, 504-507.

[16] Bloch K and Schoenheimer R. (1940) Biological precursors of creatine. J Biol Chem., 138, 167-194.

[17] J Butts, B Jacobs and M Silvis. (2018). Creatine Use in Sports. Sports Health, 10, 31-34.

[18] Close GL, Hamilton DL, Philp A, Burke LM and Morton JP. (2016). New strategies in sport nutrition to increase exercise performance. Free Radic Biol Med., 98, 144-158.

[19] Jayasena DD, Jung S and Bae YS. (2014). Changes in endogenous bioactive compounds of Korean native chicken meat at different ages and during cooking. Poult Sci., 93, 1842-1849.

[20] Yoowannakul S and Davenport A. (2018). Estimation of lean body mass by creatinine kinetics increases the prevalence of muscle wasting in peritoneal dialysis patients compared to bioimpedance. Eur J Clin Nutr., 72, 1455-1457.

[21] R Renda. (2017). Can salivary creatinine and urea levels be used to diagnose chronic kidney disease in children as accurately as serum creatinine and urea levels? A case-control study. Ren Fail., 39, 452-457.

[22] Mazze RS, Strock E, Wesley D, Borgman S, Morgan B, Bergenstal R and Cuddihy R. (2008). Characterizing glucose exposure for individuals with normal glucose tolerance using continuous glucose monitoring and ambulatory glucose profile analysis. Diabetes Technol, 10, 149-159.

[23] Suh SW, Gum ET, Hamby AM, Chan PH and Swanson RA. (2007). Hypoglycemic neuronal death is triggered by glucose reperfusion and activation of neuronal NADPH oxidase. J. Clin. Invest, 117, 910-918.

[24] Salmons S, Jarvis JC, Mayne CN, Chi MM, Manchester JK, McDougal DB. And Lowry OH. (1996). Changes in ATP, phosphocreatine, and 16 metabolites in muscle stimulated for up to 96 hours. Am J Physiol., 271, C1167-C1171.

[25] H Han, S Chae, D Hwang and J Song. (2018). Attenuating MKRN1 E3 ligase-mediated AMPK $\alpha$ suppression increases tolerance against metabolic stresses in mice Cell Stress, 2, 325-328.

[26] Toews CJ, Lowy C and Ruderman NB. (1970). The regulation of gluconeogenesis. The effect of pent-4-enoic acid on gluconeogenesis and on gluconeogenic metabolite concentrations of isolated perfused rat liver. J.Biol.Chem, $245,818-824$.

[27] Williamson JR, Browning ET and Olson NS. (1968). Interrelation between fatty acid oxidation and the control of gluconeogenesis in perfused rat liver. Advan.Enzyme Regul, 6, 67-100.

[28] Brady LJ, Armstrong NK, Muiruri KL, Romsos DR, Bergen WG and Leveille GA. (1977). Influence of prolonged fasting in the dog on glucose turnover and blood metabolites. J.Nutr, 6, 1053-1060.

[29] Goodman MN, Berger M and Ruderman NB. (1974). Glucose metabolism in rat skeletal muscle at rest. Diabetes, $23,881-888$.

[30] Hultman E and Nilsson LH. (1971). Liver glycogen in man. Effect of different diets and muscle exercise. Adv.Exp.Med.Biol, 11, 143-151.

[31] Cahill GP. (1971). Metabolic role of muscle. Adv.Exp.Med.Biol, ll, 103-109.

[32] Felig P, Posefsky T, Marliss E and Cahill GP. (1970). Alanine: key role in gluconeogenesis//Science, 167, 10031004.

[33] Krebs HA, Hems R and Lund PP. (1973). Accumulation of amino acids by the perfused rat liver in the presence of ethanol. Biochem.J, 134, 697-705. 
[34] Toews CJ, Lowy C and Ruderman NB. (1970). The regulation of gluconeogenesis. The effect of pent-4-enoic acid on gluconeogenesis and on gluconeogenic metabolite concentrations of isolated perfused rat liver. J.Biol.Chem, $245,818-824$.

[35] Williamson JR, Browning ET and Olson NS. (1968). Interrelation between fatty acid oxidation and the control of gluconeogenesis in perfused rat liver. Advan.Enzyme Regul, 6, 67-100.

[36] Marquezi ML, Roschel HA, dos Santa Costa A, Sawada LA and Lancha AH. (2003). Effect of aspartate and asparagine supplementation on fatigue determinants in intense exercise. Int. J. Sport Nutr. Exerc. Metab., 13, 65-75.

[37] Wagenmakers A. (1992). Amino acid metabolism, muscular fatigue and muscle wasting: Speculations on adaptations at high altitude. Int. J. Sports Med, 13, S110-S113.

[38] Raizel R, Leite JSM, Hypólito TM, Coqueiro AY, Newsholme P, Cruzat VF and Tirapegui J. (2016). Determination of the anti-inflammatory and cytoprotective effects of l-glutamine and l-alanine, or dipeptide, supplementation in rats submitted to resistance exercise. Br. J. Nutr, 116, 470-479.

[39] Brisola GMP and Zagatto AM. (2019). Ergogenic Effects of $\beta$-Alanine Supplementation on Different Sports Modalities: Strong Evidence or Only Incipient Findings? J Strength Cond Res., 33, 253-282.

[40] Shimomura Y, Murakami T, Nakai N, Nagasaki M and Harris RA. (2004). Exercise promotes BCAA catabolism: effects of BCAA supplementation on skeletal muscle during exercise. J Nutr. 134, 1583S-1587S.

[41] Young A. (2005). Effects on plasma glucose and lactate. Adv. Pharmacol, 52, 193-208.

[42] Shibata K and Fukuwatari T. (2013). The body vitamin B1 levels of rats fed a diet containing the minimum requirement of vitamin B1 is reduced by exercise. J Nutr Sci Vitaminol (Tokyo). 59, 87-92.

[43] Morawin B, Turowski D, Naczk M, Siatkowski I and Zembron-Lacny A. (2014). The combination of $\alpha$-lipoic acid intake with eccentric exercise modulates erythropoietin release. Biol. Sport, 31, 179-85.

[44] Nielsen FH and Lukaski HC. (2006). Update on the relationship between magnesium and exercise. Magnes. Res, $19,180-189$.

[45] Santos DA, Matias CN, Monteiro CP, Silva AM, Rocha PM, Minderico CS, Bettencourt SL and Laires MJ. (2011). Magnesium intake is associated with strength performance in elite basketball, handball and volleyball players. Magnes. Res, 24, 215-219.

[46] Beaver WL, Wasserman K and Whipp BJ. (1986). A new method for detecting anaerobic threshold by gas exchange. J. Appl. Physiol, 60, 2020-2027.

[47] Vestergaard H. (1999). Studies of gene expression and activity of hexokinase, phosphofructokinase and glycogen synthase in human skeletal muscle in states of altered insulin-stimulated glucose metabolism. Dan Med Bull., 46, 13-34.

[48] Moullé VS, Picard A, Le Foll C, Levin BE and Magnan C. (2014). Lipid sensing in the brain and regulation of energy balance. Diabetes Metab, 40, 29-33.

[49] EK Muchmejanov Nutritional composition for nourishment of obese persons and usage there of Patent Number GB2496119. Dated 22 January 2014

[50] Siemieniuk E and Skrzydlewska E. (2005). Coenzyme Q10: its biosynthesis and biological significance in animal organisms and in humans. Postepy Hig Med Dosw (Online)., 59, 150-159.

[51] Weber C, Bysted A and Hłlmer G. (1997). The coenzyme Q10 content of the average Danish diet. Int. J. Vitam. Nutr. Res, 67, 123-129.

[52] Kalén A, Appelkvist EL and Dallner G. (1989). Age-related changes in the lipid compositions of rat and human tissues. Lipids, 24, 579-584.

[53] Garrido-Maraver J, Cordero MD, Oropesa-Ávila M, Fernández Vega A, de la Mata M, Delgado Pavón A, de Miguel M, Pérez Calero C, Villanueva Paz M, Cotán D and Sánchez-Alcázar JA. (2014). Coenzyme q10 therapy. Molecular Syndromology, 5, 187-197.

[54] Goldfarb AH. (1993). Antioxidants: role of supplementation to prevent exercise-induced oxidative stress. Med Sci Sports Exerc, 25, 232-236. 
[55] Demopoulos HB, Santomier JP, Seligman ML and Pietronigro DD. (1986). Free radical pathology; rationale and toxicology of antioxidants and other supplements in sports medicine and exercise science; In sport health and nutrition; the 1984 Olympic scientific congress proceedings Champaign, 139-190.

[56] Pearson AM. (1990). Muscle growth and exercise. Crit Rev Food Sci Nutr, 29, 167-196.

[57] Urso ML and Clarkson PM. (2003). Oxidative stress, exercise, and antioxidant supplementation. Toxicol, 189, 4154.

[58] Van Klaveren RJ and Nemery B. (1999). Role of reactive oxygen species in occupational and environmental obstructive pulmonary diseases. Curr Opin Pulm Med, 5, 118-123.

[59] Kregel KC. (2002). Heat shock proteins (HSPs): modifying factors in physiological stress responses and acquired thermotolerance. J Appl Physiol, 92, 2177-2186.

[60] Moura CS, Lollo PCB, Morato PN, Risso EM and Amaya-Farfan J. (2017). Modulatory effects of arginine, glutamine and branched-chain amino acids on heat shock proteins, immunity and antioxidant response in exercised rats. Food Funct, 8, 3228-3238.

[61] Andree P, Dallner G and Ernster L. (1998). In free radicals, oxidative stress and antioxidant. Plenum Press. Ubiquinol: an endogenous lipid soluble antioxidant in animal tissues, 293-314.

[62] Ernster L. (1993). In: Active oxygens, lipid peroxides and antioxidants. CRC Press; Lipid peroxidation in biological membranes: mechanisms and implications, 1-38.

[63] Bargossi AM, Battino M, Gaddi A, Fiorella PL, Grossi G, Barozzi G, Di Giulio R, Descovich G, Sassi S and Genova ML. (1994). Exogenous CoQ10 preserves plasma ubiquinone levels in patients treated with 3-hydroxy-3methylglutaryl coenzyme a reductase inhibitors. Int J Clin Lab Res, 24, 171-176.

[64] Ernster L, Forsmark P and Nordenbrand K. (1992). The mode of action of lipid-soluble antioxidants in biological membranes: relationship between the effects of ubiquinol and vitamin $\mathrm{E}$ as inhibitors of lipid peroxidation in submitochondrial particles. J Nutr Sci Vitaminol, 548, 1-51.

[65] Ernsteer L and Dallner G. (1995). Biochemical, physiological and medical aspects of ubiquinone function. Biochim Biophys Acta, 195-204.

[66] Mataix J, Manas M, Quies J, Battino M, Cassinello M Lopez-Frias M and Huertas JR. (1997). Coenzyme Q content depends upon oxidative stress and dietary fat unsaturation. Mol Aspects Med, 18, 129-135.

[67] Gomez-Cabrera MC, Domenech E and Viña J. (2008). Moderate exercise is an antioxidant: upregulation of antioxidant genes by training. Free Radic Biol Med, 44, 126-131.

[68] Bhagavan HN and Chopra RK. (2006). Coenzyme Q10: absorption, tissue uptake, metabolism and pharmacokinetics. Free Radic Res., 40, 445-453.

[69] Linnane AW, Kios M and Vitetta L. (2007). Coenzyme Q (10) its role as a prooxidant in the formation of superoxide anion/hydrogen peroxide and the regulation of the metabolome. Mitochondrion, 7, S51-S61.

[70] Schaefer J, Navas P, Horvath Z and Jackson S. (2009). Myopathic coenzyme Q10 deficiency. Acta Myologica, 28, 41-49.

[71] Hargreaves IP. (2014). CoQ10 as a therapy for mitochondrial disease. Int. J. Biochem. Cell Biol, 49, 105-111.

[72] Kon M, Kimura F, Akimoto T, Tanabe K, Murase Y, Ikemune S and Kono I. (2007). Effect of Coenzyme Q10 supplementation on exercise-induced muscular injury of rats. Exerc Immunol Rev, 13, 76-88.

[73] Sarter B. (2002). Coenzyme Q10 and cardiovascular disease: a review. The Journal of Cardiovascular Nursing, 16, 9-20.

[74] MCR de Souza, CT Marques, CMG Dore, FRF. da Silva, HAO Rocha and EL Leite. (2007). Antioxidant activities of sulfated polysaccharides from brown and red seaweeds J Appl. Phycol, 19, 153-160.

[75] Yang L, Wang P, Wang H, Li Q, Teng H, Liu Z, Yang W, Hou L and Zou X. (2013). Fucoidan derived from Undaria pinnatifida induces apoptosis in human hepatocellular carcinoma SMMC-7721 cells via the ROS-mediated mitochondrial pathway. Mar. Drugs, 11, 1961-1976.

[76] Zapopozhets TS, Besednova NN and Loenko IN. (1995). Antibacterial and immunomodulating activity of fucoidan. Antibiot Khimioter, 40, 9-13. 
[77] Wang J, Zhang Q, Zhang Z, Zhang J and Li P. (2009). Synthesized phosphorylated and aminated derivatives of fucoidan and their potential antioxidant activity in vitro. Int J Biol Macromol, 44, 170-174.

[78] Choi JI, Raghavendran HR, Sung NY, Kim JH and Chun BS. (2010). Effect of fucoidan on aspirin-induced stomach ulceration in rats. Chem Biol Interact, 183, 249-254.

[79] Durig J, Bruhn T, Zurborn KH, Gutensohn K and Bruhn HD. (1997). Anticoagulant fucoidan fractions from Fucus vesiculosus induce platelet activation in vitro. Thromb Res, 85, 479-491.

[80] Nieman DC. (1997). Risk of upper respiratory tract infection in athletes: An epidemiologic and immunologic perspective. J. Athl. Train, 32, 344-349.

[81] S Talbott and J Talbott. (2009). Effect of BETA 1, 3/1, 6 GLUCAN on Upper Respiratory Tract Infection Symptoms and Mood State in Marathon Athletes J Sports Sci Med, 8, 509-515.

[82] Majtan J. (2012). Pleuran ( $\beta$-glucan from Pleurotus ostreatus): an effective nutritional supplement against upper respiratory tract infections? Med.Sport. Sci, 59, 57-61.

[83] Cox AJ, Pyne DB, Saunders PU and Fricker PA. (2010). Oral administration of the probiotic Lactobacillus fermentum VRI-003 and mucosal immunity in endurance athletes. British Journal of Sports Medicine, 44, 222226.

[84] Peters EM, Goetzsche JM, Grobbelaar B and Noakes TD. (1993). Vitamin C supplementation reduces the incidence of postrace symptoms of upper-respiratory-tract infection in ultramarathon runners.American Journal of Clinical Nutrition, 57, 170-174.

[85] Nieman DC, Henson DA, McMahon M, Wrieden JL, Davis JM, Murphy EA, Gross SJ, McAnulty LS and Dumke CL. (2008). Beta-glucan, immune function, and upper respiratory tract infections in athletes. Medicine and Science in Sports and Exercise, 40, 1463-1471.

[86] YM Chen, YH Tsai, TY Tsai, YS Chiu, L Wei, WC Chen and CC Huang. (2015). Fucoidan Supplementation Improves Exercise Performance and Exhibits Anti-Fatigue Action in Mice Nutrients, 7, 239-252.

\section{How to cite this article}

Emil Muchamejanov, Anatoliy Kulnasarov, Seilchan Karakov, Dias Shakenov, Victoria Belyavskaya. (2020). Nutrition base of the anti-doping products development for the athletes working efficiency encreasment. World Journal of Advanced Research and Reviews, 6(1), 218-232. 